\title{
BURNOUT EN PROFESIONALES DE ENFERMERÍA QUE TRABAJAN EN CENTROS ASISTENCIALES DE LA OCTAVA REGIÓN, CHILE.*
}

\author{
BURNOUT IN NURSING PROFESSIONALS WORKING IN HEALTH CENTERS \\ AT THE EIGHTH REGION, OF CHILE.
}

\author{
ANGÉLICA MELITA RODRÍGUEZ **, MÓNICA CRUZ PEDREROS***, \\ JOSÉ MANUEL MERINO****
}

\begin{abstract}
RESUMEN
Investigación cuantitativa, descriptiva y correlacional cuyo objetivo fue determinar la presencia de estrés laboral crónico o burnout y su probable relación con factores sociales y laborales, en los profesionales de enfermería que trabajan en Unidades de Emergencias (UE) y Servicios de Atención Médica de Urgencia (SAMU) de la Octava Región, Chile. Se trabajó con el universo conformado por 91 enfermeras(os), siendo 57 de UE y 34 de SAMU. Se utilizaron dos instrumentos recolectores de datos; el primero elaborado por la autora, que recogió información sobre variables sociodemográficas y laborales. El segundo correspondió a la Escala de Maslach Burnout Inventory (MBI); que midió el desgaste profesional producto del estrés laboral crónico y los tres aspectos del síndrome de Burnout, que corresponden a cansancio emocional, despersonalización y realización profesional. Los resultados más relevantes del estudio mostraron que más del $50 \%$ de estos profesionales corresponden a adultos jóvenes, solteros(as) y sin hijos; presentando menos de 10 años de experiencia laboral, la mayoría realiza cuarto turno. El grupo de profesionales evidenció presentar burnout en una intensidad intermedia, que estaría principalmente influenciado por variables laborales como la percepción de recursos insuficientes y la realización de exceso de turnos, al mismo tiempo el grupo de mayor edad evidenció más cansancio emocional y los viudos o separados presentaron menos despersonalización que el resto de los encuestados.
\end{abstract}

Palabras Claves: Estrés laboral crónico, Burnout, Enfermeros(as) Emergencias y SAMU.

\section{SUMMARY}

This cross sectional study is a correlational research whose principal objective was determinating the presence of chronic labour stress or burnout and its probably relationships with labour and social factors of nursing professionals working in Emergency Rooms and UMAS (Urgency Medical Atention Service) at the Eighth Region of Chile. We work with the total universe constituted by ninety-one nursing professionals: fifty-seven nurses or male nurses of emergency unities and thirty-four of UMAS. We utilized two intruments to collect the information. The first one was made by the author, and their purposes were collecting information about socio-demographical, and labors variables. The second questionnaire correspond to the Maslach Burnout Inventory (MBI) that measures emotional tiredness, dispersonalization, and professional fullfiment between nursing professionals as an outcome of stress at the working conditions. The most outstanding results of this study showed that more than $50 \%$ of nursing professional working in the crash unity are single young-adults, without children, and having not more than 10 years of work experience; most of them often working on night-turn. These ER and UMAS group of nursing professionals displayed middle-intensity burnout; the principal influence acting on this illness coming from work variables, like the perception of insufficient resources in the unities and the excess of night turns; The older group showed more emotional tiredness while separated and widowers professional presented less depersonalization than the rest of these professionals.

Keywords: chronic labor stress, burnout, nursing professionals, emergency rooms and SAMU

Fecha Recepción: 26 julio 2006 Fecha Aceptación: 21 octubre 2008

*Financiado con Proyecto DIUC 205.082.035-1.0

${ }^{* *}$ Enfermera Magíster en Enfermería. Profesor Asistente, Departamento de Enfermería Facultad de Medicina Universidad de Concepción, Concepción, Chile. Reanimadora de SAMU Hospital Las Higueras Talcahuano, Chile.E-mail: angelicar21@gmail.com

${ }^{* * *}$ Enfermera. Magíster en Enfermería, Profesor Titular, Departamento de Enfermería, Facultad de Medicina Universidad de Concepción, Chile.E-mail: mocruz@udec.cl

${ }^{* * * *} \mathrm{PhD}$ Sociology, Profesor Titular Departamento de Sociología, Facultad de Ciencias Sociales, Universidad de Concepción, Concepción, Chile.Email: jmerino@udec.cl 


\section{INTRODUCCION}

Está comprobado que las situaciones límite o extremas son las que generan mayor estrés en los individuos, ya que los someten a un estado de alerta que los prepara para enfrentar el acontecimiento que se ha percibido como amenazante.(Lazarus \& Folkman, 1986). Uno de los lugares en los que se puede encontrar escenarios que ponen a prueba el temple del ser humano, son las Unidades de Emergencia (UE), en donde a cada segundo puede ocurrir un evento en que se pone en juego la vida de las personas. A su vez, en los últimos años ha surgido otra Unidad en la cual los profesionales que trabajan en ella se ven enfrentados a situaciones extremas, esto es el Servicio de Atención Médica de Urgencia (SAMU). Se debe considerar además, que el trabajo en estas unidades, exige profesionales altamente capacitados, sin embargo, en estos lugares suceden múltiples hechos que pueden generar estrés y por lo tanto afectar el rendimiento y la capacidad laboral del individuo, lo que directa o indirectamente puede repercutir en la atención al usuario.

Actualmente, existen múltiples investigaciones que abordan la presencia de estrés laboral crónico o burnout en profesionales de Enfermería; todas ellas concluyen que la etiología es el resultado de un cúmulo de factores estresantes asociados al desempeño de la profesión y que sobrepasan los recursos de afrontamientos personales (Braham, 1999; Mingote \& Pérez, 2002; Ferrer, 2002). Por tal motivo se consideró de importancia investigar en el tema, ya que en el país existen pocos estudios que aborden esta problemática específica de los profesionales de enfermería que trabajan en las UE y SAMU de la Octava Región.

La actividad laboral es por sí misma generadora de estrés, ya que determina la exposición del empleado a ciertos estresores, que según como sean vividos, resueltos y afrontados, determinan la respuesta individual, sea adaptativa (aprendizaje, adaptación) o desadaptativa (desgaste e insatisfacción), (Carmona, Sanz \& Marín, 2002). Se han descrito varias características que influyen en la salud del trabajador, destacando: el tamaño de la empresa, la responsabilidad que se tiene, las disfunciones de rol y la falta de participación en la toma de decisiones (Mingote, 1997; Epstein, 2001). De esta manera, al relacionar estrés y trabajo, surge una muy variada cantidad de exigencias. La necesidad de satisfacción, la de autoestima y la de realización personal, cumplen un papel destacado para encontrarle sentido a la actividad que se realice (Llor \& Abad,1995). Es así como el estrés laboral se puede definir como: "El desequilibrio percibido entre las demandas profesionales y la capacidad de la persona para llevarlas a cabo" (Cruz Marín \& Vargas Fernandez, 1998). En relación con el estrés profesional, surge el termino burnout o síndrome de desgaste profesional, que describe la pérdida progresiva de energía, hasta llegar al agotamiento, síntomas de ansiedad y depresión, así como desmotivación para el trabajo, presente en trabajadores que desarrollan su actividad laboral con personas (Sánchez, 2003). Actualmente, este síndrome se define como el agotamiento emocional, despersonalización y baja realización personal. El agotamiento emocional, se refiere a la disminución y pérdida de los recursos emocionales; la despersonalización o deshumanización, consiste en el desarrollo de actitudes negativas, de insensibilidad hacia los receptores del servicio prestado y la falta de realización personal se relaciona con la tendencia a evaluar el propio trabajo de forma negativa, con vivencias de insatisfacción profesional y baja autoestima personal. Este síndrome estaría dado exclusivamente en profesionales de servicios (Maslach \& Jackson, 1981) y debe ser entendido como una respuesta al estrés laboral que aparece cuando fallan las estrategias de afrontamiento que suele emplear el profesional. El Modelo de Peiró y Salvador (Peiró \& Salvador, 1993), explica el burnout como respuesta al estrés laboral, en el cual 
una vez percibida la situación como estresante han de aparecer estrategias de afrontamiento que deben neutralizar los estresores, haciendo desaparecer las discrepancias entre demandas y recursos, puesto que la fuente de estrés persistirá en la relación cotidiana del profesional con su entorno; es decir, la situación estresora debe dejar de ser vivida como tal. En caso contrario llevarían a fracaso profesional y fracaso de las relaciones interpersonales con los sujetos receptores del servicio, provocando un sentimiento de baja realización personal en el trabajo y agotamiento emocional, que desembocaría en una actitud de despersonalización como forma de afrontamiento, que son las variables que indican la instauración del burnout. Si se mantienen en el tiempo los estresores y no se resuelve la situación, aparecerían consecuencias negativas para el individuo y la organización (Gil-Monte \& Peiró, 1997).

Estudios recientes aportan mayor información en relación a esta temática; Albadejo y Villanueva (2004), corroboraron a través de su estudio, que los profesionales que trabajan en unidades de alta demanda física y psicológica, como en las Unidades de Urgencia y Oncología, las que presentaron niveles más elevados de burnout. Sánchez-Rodríguez y De Lucas García (2001), pesquisaron que la presencia de burnout en el personal sanitario prehospitalario, correspondía principalmente a niveles intermedios a bajos. Los profesionales con hijos, los que presentaban mayor antigüedad profesional y más años dedicados a la emergencia, obtuvieron puntajes más elevados para cansancio emocional y burnout. Los profesionales que realizaban turnos de 17 horas (tarde-noche) y los que no estaban de acuerdo con su tipo de turno presentaron el puntaje más elevado para este síndrome y sus subescalas. También se pesquisó que los profesionales con problemas de relaciones interpersonales en su trabajo presentaban altos niveles de burnout y baja realización profesional. A su vez Pêra y Serra-Prat (2002) a través de su estudio pesquisaron un elevado porcentaje de este Síndrome en el personal administrativo y bajo en enfermeras. Para las escalas de cansancio emocional y despersonalización, los resultados evidenciaron alto porcentaje de burnout en los varones y sólo para cansancio emocional se observó una elevada presencia en viudos. Los de mayor edad presentaron índices más altos de burnout, los que tenía hijos evidenciaban niveles medios en la escala de cansancio emocional. Otro trabajo que entrega datos en relación a la presencia de burnout en profesionales del área de la salud es el de Hernández (2003), en donde se concluyó que el bajo reconocimiento profesional se asociaba a respuestas de estrés y de burnout. Este síndrome, afectó fundamentalmente a las mujeres médicas de la atención primaria y se detectó la presencia de síntomas de estrés en las enfermeras del mismo nivel de atención. Quiroz y Saco Méndez (2003), encontraron asociación estadística para burnout y las variables sexo masculino, profesión de médico, tiempo de servicio mayor de 12 años, baja motivación laboral, baja satisfacción laboral. A su vez se pesquisó baja asociación estadística con el trabajo mayor a 40 horas semanales, menos de 6 horas de esparcimiento a la semana, otros trabajos fuera del hospital y convivencia.

El objetivo general de este estudio fue determinar la presencia de estrés laboral crónico o burnout y su probable relación con factores sociales y laborales, en los profesionales de Enfermería que trabajan en UE y SAMU, de la Octava Región.

\section{MATERIAL Y METODO}

El presente estudio correspondió a una investigación de tipo cuantitativa, descriptiva y correlacional, que investigó la presencia de burnout en profesionales de enfermería que trabajaban en UE y SAMU, en los principales Centros Asistenciales de la Octava Región, correspondiendo estos a los Hospitales 
Base de Curanilahue, Chillán, Los Ángeles, Talcahuano y Concepción. El universo estuvo constituido por todos los profesionales de enfermería que trabajaban en estas Unidades, correspondiendo a 98 personas. La población real del estudio quedó formada por 91 profesionales de enfermería, ya que por motivos de licencia médica u otros, no se encontraban en su lugar de trabajo durante el período de aplicación de las encuestas, además 3 se negaron a responderlas, siendo en total 57 enfermeros(as) que trabajaban en UE y 34 en SAMU.

Para la aplicación de las encuestas y desarrollo del estudio se solicitó autorización a los supervisores de los Servicios involucrados en el estudio. Las encuestas fueron autoadministradas de forma voluntaria y su distribución se realizó con la colaboración de estos directivos. Para este efecto se adjuntó un consentimiento informado, el cual contempló los aspectos éticos de confidencialidad de la información atorgada, asegurando el anonimato del encuestado, además este documento explicaba de forma clara y simple los objetivos del estudio. El instrumento recolector de datos constó de un cuestionario elaborado por los investigadores que contenía preguntas en relación a variables sociodemográficas y laborales. El segundo cuestionario correspondió a la escala de Maslach Burnout Inventory (MBI), instrumento utilizado para medir estrés laboral crónico o burnout, la que entrega el estado en que el sujeto se encuentra dentro de estas tres dimensiones (alto nivel, medio y bajo). Para la validación del instrumento se utilizó la correlación de Pearson cuando se empleaban variables continuas y la correlación de Spearman's Rho cuando se empleaban variables no continuas. La confiabilidad se comprobó mediante el alfa de Cronbach. Los resultados establecieron que el instrumento MBI presentaba una elevada validez y confiabilidad, con lo que se concluye que este instrumento es seguro de utilizar.

El procesamiento de datos se efectuó en la Unidad de Estadística del Departamento de Psiquiatría de la Universidad de Concepción, mediante los programas EPI INFO $(5,0)$. SPSS 12,0 para Windows y planilla Excel. El análisis que se realizó fue principalmente descriptivo y se expresó mediante estadígrafos de tendencia central más la utilización de T de Student y ANOVA.

\section{RESULTADOS}

Del total de profesionales encuestados, un $62,6 \%$ trabajaba en UE y un $37,4 \%$ en SAMU, ubicándose en las edades comprendidas entre 22 y 39 años. En ambos lugares de trabajo las enfermeras(os) que tenían entre 22 y 29 años de edad obtuvieron porcentajes similares, correspondiendo a un $41,2 \%$ para enfermeras(os) de SAMU y de un 46,3\% para profesionales de las UE. Entre los 30 y 39 años de edad, el mayor porcentaje se ubicó en las enfermeras(os) de SAMU con un 55,9\% y un $27,8 \%$ correspondió a los profesionales de las UE.

En relación al número de hijos, se encontró que el $67,6 \%$ y el $49,1 \%$ de los enfermeras(os) de SAMU y UE respectivamente no tenían hijos. Los que dijeron tener 1 a 2 hijos correspondió al $29,4 \%$ en SAMU y a un $40,4 \%$ en UE, siendo en su mayoría (54,5\%) menores de 5 años para el grupo de enfermeras(os) SAMU. 
Tabla No 1: Antecedentes descriptivos de la muestra; profesionales de enfermería que trabajan en SAMU y Unidades de Urgencia de los principales Centros Asistenciales de la Octava Región. 2005.

\begin{tabular}{|c|c|c|c|c|c|c|}
\hline \multirow{2}{*}{ Edad } & \multicolumn{2}{|c|}{ SAMU } & \multicolumn{2}{|c|}{ Urgencia } & \multirow{2}{*}{ Totales } & \multirow{2}{*}{$\%$} \\
\hline & $\mathrm{n}$ & $\%$ & $\mathrm{n}$ & $\%$ & & \\
\hline 20 a 29 años & 14 & $41,20 \%$ & 25 & $46,30 \%$ & 39 & $44,30 \%$ \\
\hline 30 a 39 años & 19 & $55,90 \%$ & 15 & $27,80 \%$ & 34 & $38,60 \%$ \\
\hline 40 a 49 años & 1 & $2,90 \%$ & 7 & $13,00 \%$ & 8 & $9,10 \%$ \\
\hline 50 y 59 años & 0 & 0 & 7 & $13,00 \%$ & 7 & $8 \%$ \\
\hline Total & 34 & $100 \%$ & 54 & $100 \%$ & 88 & $100 \%$ \\
\hline \multirow{2}{*}{ Estado Civil } & \multicolumn{2}{|c|}{ SAMU } & \multicolumn{2}{|c|}{ Urgencia } & \multirow{2}{*}{ Totales } & \multirow{2}{*}{$\%$} \\
\hline & $\mathrm{n}$ & $\%$ & $\mathrm{n}$ & $\%$ & & \\
\hline Soltero(a) & 20 & $64,50 \%$ & 29 & $54,70 \%$ & 49 & $58,30 \%$ \\
\hline Casado(a) & 7 & $22,60 \%$ & 20 & $37,70 \%$ & 27 & $32,10 \%$ \\
\hline Viudo(a) & 0 & $0 \%$ & 1 & $1,90 \%$ & 1 & $1,20 \%$ \\
\hline Separado(a) & 3 & $9,70 \%$ & 3 & $5,70 \%$ & 6 & $7.1 \%$ \\
\hline Conviviente & 1 & $3,20 \%$ & 0 & $0,00 \%$ & 1 & $1,20 \%$ \\
\hline Total & 31 & $100,0 \%$ & 53 & $100,0 \%$ & 84 & $100,0 \%$ \\
\hline \multirow{2}{*}{$\begin{array}{c}\text { Años de } \\
\text { ejercicio de su }\end{array}$} & \multicolumn{2}{|c|}{ SAMU } & \multicolumn{2}{|c|}{ Urgencia } & \multirow{2}{*}{ Totales } & \multirow{2}{*}{$\%$} \\
\hline & $\mathrm{n}$ & $\%$ & $\mathrm{n}$ & $\%$ & & \\
\hline De 1 a 5 & 12 & $37,50 \%$ & 26 & $46,40 \%$ & 38 & $43,20 \%$ \\
\hline De 6 a 10 & 16 & $50,00 \%$ & 8 & $14,30 \%$ & 24 & $27,30 \%$ \\
\hline De 11 a 15 & 3 & $9,40 \%$ & 8 & $14,30 \%$ & 11 & $12,50 \%$ \\
\hline De 16 a 20 & 0 & $0 \%$ & 4 & $7,10 \%$ & 4 & $4,50 \%$ \\
\hline De 21 a 25 & 1 & $3,10 \%$ & 3 & $5,40 \%$ & 4 & $4,50 \%$ \\
\hline De 26 a 30 & 0 & $0 \%$ & 4 & $7,10 \%$ & 4 & $4,50 \%$ \\
\hline De 31 a 35 & 0 & $0 \%$ & 3 & $5,40 \%$ & 3 & $3,40 \%$ \\
\hline Total & 32 & $100,0 \%$ & 56 & $100 \%$ & 88 & $100 \%$ \\
\hline \multirow{2}{*}{ Tipo de turno } & \multicolumn{2}{|c|}{ SAMU } & \multicolumn{2}{|c|}{ Urgencia } & \multirow{2}{*}{ Totales } & \multirow{2}{*}{$\%$} \\
\hline & $\mathrm{n}$ & $\%$ & $\mathrm{n}$ & $\%$ & & \\
\hline Diurno & 3 & $9,10 \%$ & 6 & $11,30 \%$ & 9 & $10,50 \%$ \\
\hline Cuarto Turno & 30 & $90,90 \%$ & 39 & $73,60 \%$ & 69 & $80,20 \%$ \\
\hline $\begin{array}{l}\text { Cuarto turno } \\
\text { Modificado }\end{array}$ & 0 & $0,0 \%$ & 8 & $15,1 \%$ & 8 & $9,30 \%$ \\
\hline Total & 33 & $100,0 \%$ & 53 & $100,0 \%$ & 86 & $100,0 \%$ \\
\hline
\end{tabular}

Fuente: Investigación” Estrés laboral crónico o Burnout; en Profesionales de Enfermería que Trabajan en Unidades de Urgencia y SAMU de los Principales Centros Asistenciales de la Octava Región., VIII Región, Chile, 2005. Tesis para optar al Grado de Magíster en Enfermería, Angélica Melita Rodríguez.

En lo que respecta a años de experiencia laboral, el 87,5\% de los profesionales de SAMU poseía menos de 10 años y los que trabajaban en UE, menos de 6. El tipo de turno efectuado en ambos servicios correspondió al denominado cuarto turno, en un $90 \%$ para los de SAMU y en un 43,6\% para los de UE. En relación a los recursos que poseen en sus unidades de trabajo, el 61,8\% de las enfermeras(os) de SAMU respondió que eran suficientes, no así los que laboraban en las UE que refirieron poseer recursos insuficientes. El $50 \%$ de los profesionales de SAMU ejercían un trabajo extra, remunerado, independiente a su contrato en el hospital; en cambio, el 75,4 $\%$ de las enfermeras(os) de las UE ejercían sólo en su unidad de trabajo. Referente a ocupar cargos administrativos y de supervisión, en ambos grupos se obtuvieron porcentajes similares, con un 14, $7 \%$ para aquellos que 
trabajan en SAMU y un 15,8 \% para los de UE. En cuanto a la realización de actividades diferentes al ámbito de su profesión, como participar en grupos comunitarios u otros, el $76,5 \%$ de las enfermeras(os) de SAMU y el $68,4 \%$ de los que trabajaban en UE respondieron no realizar este tipo de actividades.
La presencia de burnout y sus subdimensiones en este grupo de profesionales fue de intensidad intermedia, con un promedio de 62.57 para la escala global de burnout y de un 19.58 para cansancio emocional, de un 8.15 para despersonalización y de un 34.85 para baja realización profesional.

Tabla No 2: Puntuaciones para la escala de MBI y sus subdimensiones, más resultados de burnout y sus subescalas, en los profesionales de enfermería que trabajan en SAMU y UE de los principales Centros Asistenciales de la Octava Región. 2005.

\begin{tabular}{|l|c|c|c|c|c|}
\hline \multicolumn{1}{|c|}{ Puntuaciones para la escala de MBI } & \multicolumn{2}{c|}{ Resultados del estudio } \\
\hline \multicolumn{1}{|c|}{ Ítems } & Niveles bajos & $\begin{array}{c}\text { Niveles } \\
\text { intermedios }\end{array}$ & $\begin{array}{c}\text { Niveles } \\
\text { elevados }\end{array}$ & $\mathrm{X}$ & $\mathrm{S}$ \\
\hline $\begin{array}{l}\text { Cansancio } \\
\text { Emocional }\end{array}$ & 1 a 18 & 19 a 26 & 27 y más & 19.56 & 10.48 \\
\hline Despersonalización & 1 a 5 & 6 a 9 & 10 y más & 8.15 & 5.40 \\
\hline $\begin{array}{l}\text { Realización } \\
\text { Profesional }\end{array}$ & 1 a 33 & 34 a 39 & 40 y más & 34.85 & 7.20 \\
\hline Burnout & 1 a 33 & 34 a 66 & 67 a 99 & 62.57 & 12.26 \\
\hline
\end{tabular}

Fuente: Investigación" Estrés laboral crónico o Burnout; en Profesionales de Enfermería que Trabajan en Unidades de Urgencia y SAMU de los Principales Centros Asistenciales de la Octava Región., VIII Región, Chile, 2005. Tesis para optar al Grado de Magíster en Enfermería, Angélica Melita Rodríguez.

El grupo de profesionales que tenía entre 50 y 59 años de edad presentaron niveles más elevados de cansancio emocional (puntaje de 29,86 ), en relación a los profesionales en otros rangos etáreos.

Si bien se pesquisó que el estado civil no influyó en la presencia de burnout, se pudo apreciar que los viudos y separados presentaron niveles bajos de despersonalización promediando 3.4 para esta subescala.

Los años de experiencia laboral no presentaron una significancia estadística en la presencia de burnout o sus subescalas, se observó que el grupo de profesionales con menor experiencia laboral presentaba puntajes más elevados para burnout (64.63), cansancio emocional (21.42) y despersonalización (9.71), que el resto de profesionales. A su vez los con mayor experiencia laboral presentaron niveles más elevados de baja realización profesional (36.61).
En cuanto al tipo de turno que realizaban y la presencia de burnout y sus subescalas, se encontró diferencia estadísticamente significativa entre los enfermeros que efectuaban un tipo de turno denominado $4^{\circ}$ turno modificado, el que corresponde a la realización de turnos adicionales al cuarto turno tradicional, en relación a los que trabajaban sólo en cuarto turno o de forma diurna exclusiva, es así como este grupo de profesionales presentó niveles más elevados para baja realización profesional, promediando 30,75 puntos en esta subescala. A su vez para cansancio emocional, sin presentar una significancia estadística, el grupo de profesionales que realizaba el cuarto turno modificado también presentó niveles más elevados en esta dimensión, que el resto de enfermeros(as) que trabajaba en otros tipos de turnos o en forma diurna, promediando para esta subescala 24 puntos.

Por otra parte, los profesionales que refirie- 
ron tener insuficientes recursos en su unidad de trabajo evidenciaron niveles elevados de cansancio emocional (correspondiendo a niveles intermedios) presentando un promedio de 22.86 puntos, y para realización profesional, promediaron el puntaje más bajo (33.28), lo que refleja una baja realización profesional; estos resultados obtuvieron gran significancia estadística; sin embargo, para la escala global de burnout y despersonalización este grupo de profesionales presentó niveles más elevados que los que refirieron una percepción de recursos suficientes en su unidad de trabajo, promediando 64.72 y 22.86 puntos, respectivamente.

Tabla No 3: Factores laborales y presencia de burnout más sus subdimenciones, en los profesionales de enfermería que trabajan en SAMU y UE de los principales Centros Asistenciales de la Octava Región. 2005.

\begin{tabular}{|c|c|c|c|c|c|}
\hline \multicolumn{2}{|c|}{$\begin{array}{l}\text { Años de Ejercicio de la } \\
\text { Profesión }\end{array}$} & \multirow{2}{*}{$\begin{array}{c}\text { Burnout } \\
64,63 \\
\end{array}$} & \multirow{2}{*}{$\begin{array}{c}\text { Cansancio } \\
\text { emocional }\end{array}$} & \multirow{2}{*}{$\begin{array}{c}\text { Despersonalización } \\
9,71 \\
\end{array}$} & \multirow{2}{*}{$\begin{array}{c}\text { Realización } \\
\text { Profesional }\end{array}$} \\
\hline Hasta 5 años & $\mathrm{x}$ & & & & \\
\hline De 6 a 10 años & $\mathrm{x}$ & 59,29 & 16,75 & 7,66 & 34,87 \\
\hline 11 y mas años & $\mathrm{x}$ & 64,46 & 21,03 & 6,8 & 36,61 \\
\hline \multirow[t]{2}{*}{ Total } & $\mathrm{x}$ & 62,79 & 19,73 & 8,06 & 34,99 \\
\hline & $\begin{array}{l}\mathrm{F} \\
\mathrm{p}\end{array}$ & $\begin{array}{l}1,737 \\
0,182 \\
\end{array}$ & $\begin{array}{l}1,719 \\
0,185\end{array}$ & $\begin{array}{l}2,585 \\
0,081 \\
\end{array}$ & $\begin{array}{r}1,43 \\
0,245 \\
\end{array}$ \\
\hline \multicolumn{2}{|c|}{ Tipo de Turno } & Burnout & $\begin{array}{l}\text { Cansancio } \\
\text { emocional }\end{array}$ & Despersonalización & $\begin{array}{l}\text { Realización } \\
\text { Profesional }\end{array}$ \\
\hline Diurno & $\mathrm{x}$ & 58,33 & 14,44 & 4,33 & 39,55 \\
\hline 4 Turno & $\mathrm{x}$ & 63,04 & 19,91 & 8,59 & 34,53 \\
\hline $\begin{array}{l}4 \text { Turno } \\
\text { modificado }\end{array}$ & $\mathrm{x}$ & 63 & 24 & 8,25 & 30,75 \\
\hline \multirow[t]{2}{*}{ Total } & $\mathrm{x}$ & 61,46 & 19,45 & 7,06 & 34,94 \\
\hline & $\begin{array}{l}\mathrm{F} \\
\mathrm{p}\end{array}$ & $\begin{array}{c}0,621 \\
0,54\end{array}$ & $\begin{array}{l}1,844 \\
0,165\end{array}$ & $\begin{array}{l}2,593 \\
0,081\end{array}$ & $\begin{array}{c}3,352 \\
0,04\end{array}$ \\
\hline \multicolumn{2}{|c|}{$\begin{array}{l}\text { Percepción de Recursos en } \\
\text { su Unidad }\end{array}$} & Burnout & $\begin{array}{l}\text { Cansancio } \\
\text { emocional }\end{array}$ & Despersonalización & $\begin{array}{l}\text { Realización } \\
\text { Profesional }\end{array}$ \\
\hline Suficiente & $\mathrm{x}$ & 59,95 & 15,53 & 7,63 & 36,78 \\
\hline Insuficiente & $\mathrm{x}$ & 64,72 & 22,86 & 8,58 & 33,28 \\
\hline \multirow[t]{2}{*}{ Total } & $\mathrm{x}$ & 62,34 & 19,20 & 8,11 & 35,03 \\
\hline & $\begin{array}{l}\mathrm{t} \\
\mathrm{p}\end{array}$ & $\begin{array}{l}3,142 \\
0,057\end{array}$ & $\begin{array}{l}4,973 \\
0,001\end{array}$ & $\begin{array}{l}1,452 \\
0,495\end{array}$ & $\begin{array}{l}1,348 \\
0,019\end{array}$ \\
\hline
\end{tabular}

Fuente: Investigación” Estrés laboral crónico o Burnout; en Profesionales de Enfermería que trabajan en Unidades de Urgencia y SAMU de los Principales Centros Asistenciales de la Octava Región., VIII Región, Chile, 2005. Tesis para optar al Grado de Magíster en Enfermería, Angélica Melita Rodríguez 
La realización de otros trabajos extra remunerados, el ejercicio de algún cargo de coordinación y la práctica de actividades diferentes a lo profesional o participación en grupos de la comunidad no presentaron relación estadísticamente significativa en la presencia de Burnout y sus subescalas.

\section{DISCUSION}

Los hallazgos de este estudio mostraron que el número de profesionales de enfermería que trabaja en UE y SAMU de los principales centros hospitalarios de la Octava Región son insuficientes en relación a la alta densidad de población que les corresponde atender, siendo la Octava Región la segunda con mayor población, 1853678 habitantes y 50 hab $/ \mathrm{km}^{2}$, después del área metropolitana, representando alrededor del $13 \%$ de la población según censo de 2002 (INE, 2006). Esto lleva consigo una mayor carga laboral con jornadas de más de 8 horas diarias, con turnos rotatorios con la consecuente alteración del biorritmo, trato con pacientes críticos y unidos a recursos insuficientes, constituyendo éstos factores de riesgo en la aparición de síndrome de burnout.

En cuanto al perfil sociodemográfico, el total de profesionales encuestados se caracterizó por ser adultos jóvenes predominantemente solteros y sin hijos. Al comparar la edad de estos profesionales con los que trabajan en el SAMUR de España (Sánchez-Rodríguez \& De Lucas García, 2001), se observó que acá también predominan la edades entre 30 y 39 años pero sin hijos, pero a diferencia de los enfermeros de nuestra Región, en su mayoría son casados.

En cuanto a los principales aspectos laborales se encontró que los enfermeros(as) que trabajan en estas unidades de choque llevan ejerciendo menos de 10 años, lo que puede estar relacionado directamente con los años que tiene el SAMU en nuestra Región, ya que es un servicio relativamente nuevo. A su vez, se observó que en las Unidades de Emergencia los profesionales de enfermería que se encontraban trabajando en ellas, tienen menos de 6 años de experiencia laboral. Ambos fenómenos estarían íntimamente relacionados con la edad que presentan estos profesionales, ya que la gran proporción corresponde a adultos jóvenes. Sánchez-Rodríguez y De Lucas García (2001), a través de su estudio revelan que los profesionales que trabajan en este tipo de unidades de emergencia en España, presentan un promedio de antigüedad en su profesión de 8.9 años, específicamente en unidades de emergencia 6.8 años, y trabajando en el SAMUR un promedio de 4.1 años; realidad que se asemeja a la situación que se da en nuestra región. Las horas trabajadas por el grupo de enfermeros que participó en esta investigación se distribuyen, principalmente en el denominado cuarto turno que consiste en 12 horas continuadas de trabajo diurno, al día siguiente se realizan 12 hrs. de noche disponiendo finalmente de dos días libres. En este aspecto, el trabajo de SánchezRodríguez y De Lucas García (2001), también muestra que mayoritariamente los profesionales del SAMUR realizan un tipo de turno similar, pero de 17 horas llamado mañanatarde; otro estudio realizado en Cuzco, Perú; revela que las enfermeros(as) realizan como promedio 36.9 horas semanales, siendo lo mínimo 36 horas y lo máximo 60 horas. Albadejo y Villanueva (2004), encontraron que los profesionales mayoritariamente realizaban turnos rotatorios que se distribuían entre mañana, tarde y noche, detectando en bajo porcentaje la realización de turnos continuados de 12 y hasta 24 horas.

En relación a la presencia del síndrome de burnout en el grupo de profesionales estudiados, se encontró que está presente en una intensidad intermedia; a su vez, se detectó cansancio emocional, despersonalización y realización profesional en niveles intermedios. Es decir este grupo no presenta niveles significativamente elevados, por lo que podemos interpretar que al existir presencia 
de burnout y de sus subescalas de cansancio emocional y despersonalización, se genera en forma inversa un nivel poco óptimo de realización profesional. Datos similares aporta el estudio de Albadejo y Villanueva (2004), quienes encontraron que entre enfermeros(as) que trabajaban en diferentes unidades dentro de un hospital en Madrid España, los que presentaban mayor nivel de burnout, agotamiento emocional y despersonalización, correspondía a los que trabajaban en las unidades de Urgencias y Oncología.

En cuanto a los factores relacionados con la presencia de burnout, se encontró que los sociodemográficos presentan un bajo impacto en este síndrome, destacándose la edad, ya que los profesionales de mayor edad evidenciaron presentar niveles más elevados de cansancio emocional, situación similar se manifiesta en los resultados del estudio realizado por Pera y Serra-Prat M (2002), el que revela que la edad se correlacionaba débilmente con la escala de cansancio emocional; sin embargo, los de mayor edad eran los que presentaron índices superiores de burnout. Otra variable que presentó cierta influencia en la presencia de las subdimensiones de burnout fue el estado civil o presencia de pareja, según los resultados obtenidos, los profesionales solteros y casados presentaron índices más elevados de despersonalización que los viudos y separados, a su vez los solteros evidenciaron una tendencia a niveles bajos de realización profesional. La investigación de Álvarez y Arce (2005), revela que la despersonalización y el cansancio emocional predominaba entre los profesionales que se encontraban con pareja; realidad que en parte se asemeja a la de los enfermeros(as) de estas unidades de choque, ya que de igual manera los que estaban casados presentaron puntuaciones más elevadas para despersonalización que los viudos y separados. Dicho fenómeno podría estar influenciado a su vez por la edad, ya que los profesionales más jóvenes y con menor experiencia laboral corresponden a personas solteras o recientemente casadas, y por lo cual, se ven enfrentados a múltiples situaciones que son nuevas para ellos generando estrés que se refleja en la presencia de cansancio emocional o baja realización profesional. En cuanto a los años de experiencia laboral o antigüedad laboral, no se encontró en este estudio relación estadísticamente significativa para la presencia de burnout y sus subescalas, situación similar se detectó en el estudio de Pera y Serra-Prat (2002); quienes identificaron que los años de ejercicio profesional no se relacionaban con cambios en la escala de MBI. Sin embargo, existe evidencia en otras investigaciones que después de 10 años de antigüedad laboral, puede existir un periodo de sensibilización a partir del cual el profesional sería especialmente vulnerable a la aparición de este síndrome (Artiga, 2000; Albadejo \& Villanueva, 2004). Información similar entrega el trabajo de Quiroz y Saco Méndez (2003), quienes detectaron una fuerte asociación entre un tiempo de ejercicio laboral mayor a 12 años y la presencia de burnout.

Dentro de los factores relacionados con el ámbito laboral y que repercutieron en la presencia de este síndrome y sus subescalas fue la realización de un tipo de turno denominado cuarto turno modificado, en que el profesional, además de su cuarto turno debe realizar otros extras o trabajar horas adicionales al cuarto turno preestablecido. En relación a este punto existen antecedentes que revelan que la turnicidad somete al profesional a continuos cambios que le impiden la instauración de hábitos saludables y a su vez altera el mantenimiento de relaciones interpersonales (Gil-Monte \& Peiró, 1997; Álvarez \& Arce, 2005); al mismo tiempo este sistema produce alteración del biorritmo, lo cual incide directamente en la presencia de burnout, a su vez, el cansancio mental de los enfermeros(as) al trabajar de noche puede conllevar a una disminución de sus capacidades mentales, con disminución del rendimiento laboral (Mingote \& Pérez, 2002). Otro estudio que detectó este fenómeno fue 
el de Sánchez-Rodríguez y De Lucas García (2001), el que identificó que los profesionales que realizaban un turno de 17 horas que correspondía a una tarde más la noche, presentaban los puntajes más altos para burnout y sus subescalas. El aspecto laboral que incidió fuertemente en la presencia de las subdimensiones de este síndrome fue la percepción de recursos insuficientes en la unidad en la cual trabajan, es decir, la percepción de que los recursos con los cuales se cuenta para desarrollar las actividades diarias dentro de la unidad son insuficientes, estarían influyendo de manera directa en la presencia de cansancio emocional y más intensamente en la presencia de una baja realización profesional. Si bien, no se encontraron estudios que abordaran específicamente este aspecto, la investigación de Quiroz y Saco Mendez (2003), establece una relación directa entre la falta de motivación y la presencia de burnout. En relación a esto, detectó que el 92\% de los encuestados referían que era de suma importancia que los profesionales se desenvolvieran en buenas condiciones de trabajo, con comodidad y avances tecnológicos acorde a las funciones que desarrollaban, junto con ello expresaban la importancia de la capacitación permanente en función de las necesidades de sus trabajos. Con estos resultados se hace necesario dotar de recursos suficientes las unidades de trabajo de estos profesionales para propiciar el rendimiento adecuado de sus funciones.

Por último, cabe concluir que los profesionales de enfermería que trabajan en las UE y SAMU, unidades de choque, evidencian una tendencia a presentar estrés laboral crónico o Burnout, lo que los convierte en un grupo vulnerable dentro del ámbito asistencial, con tendencias a manifestar alteraciones físicas y psicológicas asociadas a este síndrome, lo que debe generar una inquietud en las autoridades de salud respectivas, para la formulación y aplicación de políticas de prevención primaria y secundaria. Además no deja de ser relevante la presencia de nive- les medianamente elevados de despersonalización, ya que al ser profesionales que trabajan directamente con personas y que al estar relacionados con el dolor, sufrimiento del cuerpo y el alma, no pueden estar completamente ajenos de esta situación al punto que podría repercutir en la calidad de la atención que están entregando. Por otra parte, la presencia de moderada realización profesional, en trabajadores que se encuentran altamente capacitados, también genera una inquietud para averiguar los factores que estarían influyendo directamente en la aparición de este fenómeno. En relación a este último punto, la investigación realizada por Hernández (2003), entrega información en relación a que el bajo reconocimiento que percibían los enfermeros por su trabajo realizado se asociaba a la presencia de burnout, por lo tanto a baja realización profesional y a respuestas de estrés.

\section{REFERENCIAS BIBLIOGRAFICAS}

Albadejo, R.\& Villanueva, R. (2004). Síndrome de Burnout en el personal de enfermería de un hospital de Madrid. Revista Española de Salud Pública.78 (004), 505-516.

Álvarez, A. \& Arce, M. (2005) Síndrome de Burnout en médicos de hospitales públicos de la ciudad de Corrientes. Revista de Postgrado de la VI Cátedra de Medicina, enero (141), 27-30. Argentina.

Artiga, A. (2000) La enfermería ante el Síndrome Burnout. Estudio comparativo entre atención primaria y atención especializada. Revista Enfermería Científica, jul-ago; 220(221),11-16.

Braham, B. (1999). Como controlar el estrés y mantener la calma en situaciones difíciles. Madrid, España. Ediciones Time Mirror de España S.A.

Cruz Marin, C. \& Vargas Fernandez, L. (1998). Estrés, entenderlo es manejarlo (2da. Edición). Santiago, Chile. Ediciones Universidad Católica de Chile.

Carmona, F., Sanz, L. \& Marín, D. (2002) Relaciones entre el estrés en profesionales de enfermería. Factores sociodemográficos y reac- 
tividad al estrés. Revista Enfermería Científica. ene-feb, 238(239) 33-39.

Chile. Instituto de Normalización Estadística (INE). (2006).

Epstein, R. (2001) Comunicación, burnout y resultados clínicos: más preguntas que respuestas. Atención Primaria. 27 (7) 511-513.

Ferrer, R., (2002) Burnout o síndrome de desgaste profesional. Revista de medicina clínica. 119, (13), 495-496.

Gil-Monte, R. \& Peiro, J. M. (1997). Desgaste psíquico en el trabajo: El síndrome de quemarse. Madrid. Síntesis S.A.

Hernández, J. (2003). Estrés y Burnout en profesionales de la salud de los niveles primario y secundario de atención. Revista Cubana de Salud Pública, 29 (002), 103-110.

Lazarus, R. \& Folkman, S. (1986). Estrés y procesos cognitivos, Barcelona, Ediciones Martínez de Roca.

LLor, B. \& Abad, M. . (1995). Ciencias psicosociales aplicadas a la salud. Madrid.

McGraw-hill-Interamericana de España.

Maslach, C. \& Jackson, S. (1981) The measurement of experienced burnout. J Occup Psychol, (2), 99-113.
Mingote, J. (1997). Síndrome de desgaste profesional (Burnout). Medicina y Seguridad del Trabajo.4(174), 6-71

Mingote, J. \& Pérez, S. (2002). Estrés en la Enfermería. El cuidado del cuidador,Madrid, Ediciones Díaz de Santos.

Peiro, J. M. \& Salvador, A. (1993). Control del estrés laboral. Madrid. Eudema.

Pera, G. \& Serra-Prat; M. (2002). Prevalencia del síndrome del quemado y estudio de los factores asociados en los trabajadores de un hospital comarcal. Gaceta Sanitaria. 16 (6), 480-486.

Quiroz V, R. \& Saco Méndez, S. (2003). Factores asociados al síndrome de burnout en médicos y enfermeras del hospital Nacional de Essalud del Cusco. SITUA. Revista semestral de la Facultad de Medicina Humana. 12(23),11-22.

Sánchez -Rodríguez, A. \& De Lucas García; N. (2001). Estrés laboral en el profesional de un servicio de emergencia prehospitalario. Emergencias. (13), 170-175.

Sánchez, A. (2003). Estrés, pórtico de la depresión. (1ºd.) Madrid. Editorial San Pablo. 